\title{
Reflexos das relações interpessoais na participação do controller em decisões organizacionais
}

\author{
Heitor Gerson dos Santos \\ UFSC - Universidade Federal de Santa Catarina \\ heitorg.santos@hotmail.com \\ Joice Denise Schafer \\ UFSC - Universidade Federal de Santa Catarina \\ schafer.joice@gmail.com \\ Rogério João Lunkes \\ UFSC - Universidade Federal de Santa Catarina \\ rogeriolunkes@hotmail.com
}

\section{Resumo}

Este estudo tem como objetivo analisar a influência das relações interpessoais na participação do controller na tomada de decisões organizacionais (operacionais e estratégicas). 0 estudo enquadra-se como descritivo, com abordagem quantitativa. Os dados foram coletados por meio de questionários aplicados junto aos controllers de empresas brasileiras. Os respondentes da pesquisa (42), em sua maioria, são profissionais formados em Ciências Contábeis, com idade superior a 35 anos e com mais de cinco anos de experiência na função e na empresa as quais estão vinculados atualmente. Os resultados da pesquisa permitem inferir que embora 0 relacionamento interpessoal seja estatisticamente significativo para 0 envolvimento do profissional da controladoria na tomada de decisão operacional, não parece ser determinante para o envolvimento do controller na tomada de decisão estratégica das organizações. O tempo de experiência na função desmontou como a variável de controle com maior efeito sobre o envolvimento do profissional em decisões estratégicas, enquanto o gênero se mostrou relevante tanto em nível estratégico quanto operacional. Controllers do gênero masculino têm maior nível de participação na tomada de decisão organizacional. Estudos que envolvam variáveis organizacionais, ambientais e mesmo características pessoais da alta gestão são sugeridos para pesquisas futuras que busquem identificar fatores relacionados ao envolvimento do profissional da controladoria em diferentes níveis de tomada de decisão organizacional.

Palavras-chave: Controller. Relações interpessoais. tomada de decisão organizacional.

ISSN: $1984-6266$

Recebimento:

$14 / 08 / 2020$

Aprovação:

03/01/2021

Editor responsável pela aprovação do artigo:

Dra. Nayane Thays Krespi Musial

Editor responsável pela edição do artigo:

Dra. Luciana Klein

Avaliado pelo sistema:

Double Blind Review

A reprodução dos artigos, total ou parcial, pode ser feita desde que citada a fonte.

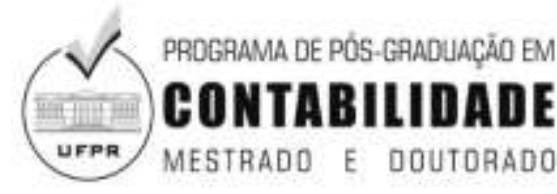

DOI:

http://dx.doi.org/10.5380/rcc.v12i2.75842 


\title{
REFLECTIONS OF INTERPERSONAL RELATIONS IN THE CONTROLLER'S PARTICIPATION IN ORGANIZATIONAL DECISIONS
}

\begin{abstract}
This study aims to analyze the reflexes of interpersonal relationships in the controller's participation in making organizational decisions (operational and strategic). The study is classified as descriptive, with a quantitative approach. The data were collected through questionnaires applied to the controllers of Brazilian companies. Most of the survey respondents (42) are professionals graduated in Accounting Sciences, over the age of 35 years and with more than five years of experience in the role and in the company to which they are currently linked. The results of the research allow us to infer that although the interpersonal relationship is statistically significant for the involvement of the controllership professional in making operational decisions, it does not seem to be decisive for the involvement of the controller in the strategic decision making of organizations. The time of experience in the function dismantled as the control variable with the greatest effect on the professional's involvement in strategic decisions, while gender was relevant both at the strategic and operational levels. Male controllers have a higher level of participation in organizational decision-making. Studies involving organizational, environmental variables and even personal characteristics of senior management are suggested for future research that seeks to identify factors related to the involvement of the controllership professional at different levels of organizational decision-making.
\end{abstract}

Keywords: Controller. Interpersonal relationships. Organizational decision making.

\section{Introdução}

As funções do controller têm sido amplamente discutidas na literatura, assim como no desenvolvimento de sua prática profissional. De um lado o controller é entendido como o responsável pelos relatórios gerenciais, ou seja, pelas informações que dão base a tomada de decisões estratégicas nas organizações, por outro lado, o controller é entendido como o profissional responsável por fornecer informações aos gerentes de nível médio, apoiar o desenvolvimento do planejamento das áreas, motivar os colaboradores, controlar custos, monitorar 0 andamento de indicadores, entre outras funções (Rouwelaar, 2007; Weber, 2011). Em outras palavras, 0 profissional da controladoria pode estar envolvido em atividades que são base para a tomada de decisão em nível operacional ou estratégico.

Ainda não há um consenso sobre esta questão na literatura, mas as discussões vêm avançando, e demonstram haver diferentes níveis de envolvimento dos controllers nas atividades das empresas. $E$ que ocorrem de acordo com o que é desejável e benéfico para as organizações (Maas \& Matejka, 2009), e de interesse dos próprios profissionais (Pierce \& O'Dea, 2003). De acordo com Rouwelaar, Bots e De Loo (2018), alguns dos elementos que vem sendo apontados por estudos anteriores como relevantes no processo de envolvimento dos controllers na tomada de decisão incluem o relacionamento interpessoal do profissional, as características organizacionais e as características ambientais. Enquanto as duas últimas características estão relacionadas com aspectos macroorganizacionais, tais como a situação financeira de uma organização e a pressão dos concorrentes, ou questões estratégicas que devam ser consideradas, o relacionamento interpessoal está diretamente ligado as características pessoais do controller. 
Neste sentido, a forma como o controller se relaciona com sua equipe e com a alta gestão da organização pode ser decisiva para o seu grau de envolvimento na tomada de decisão organizacional. Wolf, Weibenberger, Wehner e Kabst (2015) destacam a relevância do entendimento acerca da participação do controlador nas decisões, juntamente com suas próprias motivações e as expectativas dos outros. Nesse sentido, a literatura evidencia que os estudos sobre o papel dos controllers têm foco na mudança do papel desse ator organizacional, mas há uma lacuna na compreensão dos motivadores desta mudança em uma perspectiva micro organizacional, ou seja, na maneira como os profissionais interagem com outros atores (Lavarda, Scussel \& Schäfer, 2018). Assim, este estudo tem como objetivo analisar a influência das relações interpessoais na participação do controller na tomada de decisões organizacionais, em nível operacional e estratégico.

Este estudo justifica-se, do ponto de vista teórico, pela contribuição à literatura de controladoria, ao dar continuidade a linha de pesquisas de Rouwelaar et al. (2018), que indicam características pessoais dos controllers como determinantes para a participação do profissional na tomada de decisão organizacional. No que se refere as contribuições gerenciais, chama-se a atenção para a o fato de que para que o controller seja convidado a envolver-se mais com a tomada de decisão, ele necessitará, além das informações e conhecimento adequado, características pessoais específicas, que inspirem a confiança dos demais gestores.

Como implicações dos resultados, é possível inferir que, embora um bom relacionamento entre o controller e os gestores das empresas contribua para uma maior participação do profissional nas tomadas de decisões operacionais, quando se trata de decisões estratégicas, o bom relacionamento entre as partes tende a não ser decisivo para o envolvimento do controller na tomada de decisão. Neste caso, outras variáveis, como experiência do funcionário na empresa, parecem ter maior relevância.

\section{Referencial Teórico e hipóteses}

A controladoria é uma área em crescimento tanto em termos de possibilidades de trabalho nas organizações (Gomes, Souza \& Lunkes, 2014; Schnorrenberger, Lunkes \& Castro, 2015; Gomes, Lunkes \& Schnorrenberger, 2015; Massocco, Schäfer \& Lunkes, 2016), quanto em publicações especializadas (Lunkes, Schnorrenberger, Souza, \& Rosa, 2012; Marostega, Lyrio, Lunkes, \& Schnorrenberger, 2014), disciplinas acadêmicas e grupos de pesquisa (Lunkes et al., 2012).

Entre os temas de pesquisa da controladoria está a definição das funções básicas, ou seja, orientar o campo de atuação da área de conhecimento (Lunkes, Schnorrenberger, \& Rosa, 2013). Neste sentido, nas últimas décadas diversos estudos foram desenvolvidos em países, como Brasil (Siqueira \& Soltelinho, 2001; Lunkes, Schnorrenberger, Gasparetto \& Vicente 2009; Oro, Dittadi, Carpes \& Benoit, 2009; Souza \& Borinelli, 2009; Ferrari, Cunha, Lunkes \& Borgert, 2013; Gomes, Souza \& Lunkes, 2014) Estados Unidos (Lunkes et al.; 2009), Alemanha (Lunkes et al.; 2009) e Espanha (Schnorrenberger Castro \& Lunkes, 2015), buscando identificar quais as funcionalidades atribuídas aos controllers nestes países, bem como o perfil exigido destes profissionais pelos empregadores.

De acordo com Goretzki, Strauss e Weber (2013), desde os anos 1990 o papel do controller nas organizações vem passando por mudanças. Os autores afirmam que o profissional vem desenvolvendo cada vez mais atividades em nível estratégico, demonstrando capacidade de adicionar valor aos negócios, passando a ser percebido, assim, como "parceiros de negócios" pela alta administração. No entanto, estudos têm demonstrado que esta mudança não é universal (Järvenpää, 2007; Lunkes, Schnorrenberger, Gasparetto \& Vicente, 2009). Neste sentido, a pesquisa de Rouwelaar, Bots e De Loo (2018), aplicada a 119 controllers de unidades de negócios, de 77 diferentes empresas, aponta que os controllers das unidades tendem a influenciar as decisões operacionais tomadas pelos gerentes, porém em poucos casos os controllers influenciam os gerentes quando as decisões são estratégicas. 
Mediante tal falta de consenso sobre o envolvimento do controller na tomada de decisão, Szukits (2019) defende que as atribuições gerais a respeito do trabalho a ser desenvolvido pelo profissional tendem a serem estabelecidas de acordo com as necessidades das organizações e o entendimento da alta gestão sobre 0 trabalho que precisa ser desenvolvido. Karlsson (2019) acredita que os controllers podem moldar as tarefas a eles atribuídas a partir de suas habilidades específicas, muitas vezes provenientes de características pessoais ou relacionamentos interpessoais.

As relações interpessoais permitem, em geral, troca de experiências, de conhecimento técnico e teórico, que são responsáveis pelo aprimoramento do profissional (Fernandes et al., 2015). Weber (2011) destaca que a interação entre controllers e gerentes é relevante para ambos. $O$ autor destaca que 0 controller deve conhecer a personalidade individual de cada gestor, para ser preciso e objetivo no processo de transferência de informação, no sentido de permitir que gestor compreenda adequadamente a informação recebida e consiga, a partir dela, tomar decisões eficientes. Com base nestes argumentos, propõem-se as seguintes hipóteses de pesquisa:

H1: O relacionamento interpessoal do controller afeta positivamente o nível de envolvimento do profissional na tomada de decisões operacionais.

$\mathrm{H}$ 2: $\mathrm{O}$ relacionamento interpessoal do controller afeta positivamente o nível de envolvimento do profissional na tomada de decisões estratégicas.

Um conflito pessoal entre o mensageiro da informação, neste caso o controller, e o leitor da mesma, os gestores, pode levar à uma tomada de decisão equivocada (Fernandes et al., 2015). O relacionamento entre os indivíduos influencia diretamente a organização, principalmente, quando informações levam a decisões que impactam decisões estratégicas. Desta forma, os gestores tendem a evitar a participação de indivíduos com maiores dificuldades de relacionamento interpessoal na tomada de decisão de nível estratégico, embora sua participação seja comum na tomada de decisão operacional (Rouwelaar, Bots \& Vanamelsfort, 2008).

$O$ choque de personalidades dentro de uma organização pode promover conflitos, porém o diferencial está em converter essas desavenças em crescimentos (Fernandes et al., 2015). Pontos de fortalecimentos, no final podem mudar o posicionamento de uma organização perante dificuldades, tornando-a uma empresa com uma capacidade diferenciada de contornar problemas com mais facilidade.

\section{Procedimentos Metodológicos}

Esta pesquisa tem como objetivo analisar a influência das relações interpessoais na participação do controller na tomada de decisões organizacionais (operacionais e estratégicas). $O$ estudo pode ser classificado como descritivo, por descrever a relação entre as variáveis relacionamento interpessoal e envolvimento do controller da tomada de decisão estratégica e operacional. Quanto à abordagem do problema, utilizou-se da pesquisa quantitativa, já que os dados analisados são numéricos, sendo a estatística utilizada para analisar os resultados e responder ao problema de pesquisa proposto.

Com relação aos procedimentos adotados para o desenvolvimento da pesquisa, utilizou-se o levantamento (ou survey), utilizando-se de questionários para a coleta de dados. Este foi estruturado em 3 blocos principais, conforme demonstrado no Quadro 1. Para os Blocos 1 e 2, os respondentes foram orientados a ler as afirmativas atentamente e na sequência indicar em que medida concordavam ou discordavam com cada uma delas, utilizando: 1 para discordo totalmente, 2 para discordo pouco, 3 para nem concordo, nem em discordo, 4 para concordo pouco, e 5 para concordo totalmente. A escala Likert de 5 pontos foi utilizada, seguindo a estrutura do estudo base de Rouwelaar, Bots \& De Loo (2018). 
Quadro 1: Estrutura do Questionário

\begin{tabular}{|c|c|c|c|}
\hline Bloco & Variáveis & Autores base & $\mathbf{N}^{0}$ de questões \\
\hline 1: Relacionamento interpessoal & $\begin{array}{c}\text { Trabalho em equipe; confiança nos } \\
\text { superiores e subordinados; } \\
\text { relacionamento com superiores e } \\
\text { subordinados. }\end{array}$ & $\begin{array}{c}\text { Morgeson, Reider \& } \\
\text { Campion (2005); } \\
\text { Tzafrir e Eitam-Meilik } \\
\text { (2005). }\end{array}$ & 5 \\
\hline 2: Envolvimento na tomada de \\
decisão & $\begin{array}{c}\text { Decisões estratégicas e decisões } \\
\text { operacionais. }\end{array}$ & $\begin{array}{c}\text { Rouwelaar, Bots \& } \\
\text { De Loo (2018) }\end{array}$ & 7 \\
\hline 3: Perfil dos respondentes & $\begin{array}{c}\text { Idade, gênero, área de formação e } \\
\text { experiência. }\end{array}$ & - & 6 \\
\hline
\end{tabular}

Os questionários foram enviados para 259 profissionais da área da controladoria das empresas listadas no Catálogo de Exportadores Brasileiros (CEB) da CNI. Esta listagem de e-mails foi disponibilizada pelas empresas durante contato prévio feito pelos pesquisadores com cada uma das empresas do CEB. Dentre os 259 profissionais contatados, 40 (15,4\%) responderam à pesquisa, encaminhada em Formulário Google, por email, no mês de julho de 2019.

Para análise dos dados utilizou-se estatística descritiva e a técnica de modelagem de equações estruturais (Structural Equation Modeling - SEM). Segundo Bido, Silva, Souza e Godoy (2010), a SEM pode ser classificada em dois tipos: a) Modelagem de Equações Estruturais baseada em covariâncias (MEEBC) ou simplesmente modelos LISREL; e b) Mínimos Quadrados Parciais, que realiza a modelagem de equações estruturais por partes. Recentemente este último tem sido denominado de Partial Least Squares (PLS). O PLS pode ser usado tanto para predição, como para a construção de modelos teóricos. A opção pelo PLS deve-se ao fato de tal método permitir trabalhar com amostras pequenas, não exigir que os dados apresentem distribuição normal e ser capaz de lidar com alta complexidade (Bido et al., 2010). Ele consiste de um sistema de relações lineares entre variáveis latentes que é resolvido por partes (combinação de constructos teóricos e medidas), uma de cada vez (Bido et al., 2010). O PLS é uma técnica de estimação de regressão linear, baseada na decomposição das matrizes de variáveis e de covariáveis.

Antes da aplicação do PLS realizaram-se testes para validação e adequação do modelo e análises de relações entre os constructos. Após isso, os dados foram migrados para o software ADANCO para as análises do modelo de mensuração e do modelo estrutural. A base de dados foi salva no formato Excel, convertida para o formato CSV, e executada no software ADANCO, a fim de aplicar a técnica de Mínimos Quadrados Parciais.

\section{Análise dos Dados}

\subsection{Análise descritiva}

Na presente seção são apresentados os dados demográficos e a estatística descritiva referente aos respondentes. Participaram da pesquisa, 40 controllers de empresas brasileiras, na Tabela 1, apresentam-se os dados demográficos dos respondentes da pesquisa, dentre os quais se destacam gênero, idade, formação e experiência dos profissionais.

Considerando os dados da Tabela 1, percebe-se um maior número de respondentes do sexo masculino (70\%), enquanto a predominância do curso de formação é em Ciências Contábeis (69\%). Cabe destacar, ainda, que dois dos respondentes informaram mais de uma área de formação, totalizando assim 42 cursos de graduação completados pelos 40 respondentes. A área de formação classificada na Tabela 1 como "outros", se refere aos cursos de direito, economia e área da saúde. Quando analisada a idade dos indivíduos, chama a atenção o fato de nenhum dos respondentes ter idade inferior a 30 anos, demonstrando a tendência do 
controller ser um profissional com maior experiência, o que se confirma quando analisados os dados de tempo de experiência na empresa e tempo de experiência na função. Dentre os respondentes somente $22 \%$ (9) possuem menos 5 anos ou menos de experiência na função de controller, enquanto apenas $7 \%$ informaram trabalhar na empresa a 5 anos ou menos. De forma geral, portanto, a amostra desta pesquisa se caracteriza por controllers, predominantemente do sexo masculino, formados em ciências contábeis, acima de 30 anos e com um alto nível de experiência na função e na empresa onde trabalham.

Tabela 1: Dados Demográficos

\begin{tabular}{|c|c|c|c|c|c|c|}
\hline Gênero & Quant. & $\%$ & & Formação & Quant. & $\%$ \\
\hline Masculino & 28 & $70 \%$ & & Administração & 7 & $17 \%$ \\
\hline Feminino & 12 & $30 \%$ & & Ciências Contábeis & 29 & $69 \%$ \\
\hline TOTAL & 40 & $100 \%$ & & Outros & 6 & $14 \%$ \\
\hline Temp. Experiência & Quant. & $\%$ & & TOTAL & 42 & 1 \\
\hline Até 5 anos & 9 & $23 \%$ & & Temp. Empresa & Quant. & $\%$ \\
\hline De 6 a 10 anos & 11 & $28 \%$ & & Até 5 anos & 4 & $10 \%$ \\
\hline De 11 a 15 anos & 7 & $18 \%$ & & De 6 a 10 anos & 16 & $40 \%$ \\
\hline De 16 a 20 anos & 2 & $5 \%$ & & De 11 a 15 anos & 8 & $20 \%$ \\
\hline De 21 a 25 anos & 8 & $20 \%$ & & De 16 a 20 anos & 6 & $15 \%$ \\
\hline Acima de 25 anos & 3 & $8 \%$ & & Acima de 20 anos & 6 & $15 \%$ \\
\hline \multirow[t]{9}{*}{ TOTAL } & 40 & $100 \%$ & & TOTAL & 40 & $100 \%$ \\
\hline & & Idade & Quant. & $\%$ & & \\
\hline & & Até 35 anos & 3 & $8 \%$ & & \\
\hline & & De 36 a 40 anos & 11 & $28 \%$ & & \\
\hline & & De 41 a 45 anos & 9 & $23 \%$ & & \\
\hline & & De 46 a 50 anos & 6 & $15 \%$ & & \\
\hline & & De 51 a 55 anos & 9 & $23 \%$ & & \\
\hline & & Acima de 56 anos & 2 & $5 \%$ & & \\
\hline & & TOTAL & 40 & $100 \%$ & & \\
\hline
\end{tabular}

Os resultados referentes a estatística descritiva, especificamente média e desvio padrão dos constructos, são apresentados na Tabela 2. 
Tabela 2: Estatística Descritiva

\begin{tabular}{ccc}
\hline Variáveis & Média & Desvio Padrão \\
\hline RI & 4,46875 & 0,6997023 \\
\hline DOP & 4,13333 & 1,0202433 \\
\hline DES & 3,45 & 1,2288548
\end{tabular}

Rl: Relacionamento Interpessoal; DOP: Decisão Operacional; DES: Decisão Estratégica.

Fonte: Dados da Pesquisa

A média de respostas para o relacionamento interpessoal foi a mais alta entre as variáveis analisadas, de 4,47, enquanto o desvio padrão foi o menor, o que significa dizer que os respondentes possuem um bom nível de relacionamento interpessoal (próximo do valor máximo, 5), e que não houve uma grande variação entre as respostas apresentadas pelos profissionais. No que se refere a participação do controller em decisões operacionais a média foi de 4,13 , demonstrando um alto nível de participação dos controllers, no entanto, com um desvio padrão acima de 1 , demonstrando uma variação maior se comparado a variável relacionamento interpessoal. Como esperado, a participação do controller em decisões estratégicas apresentou uma média inferior e um desvio padrão superior, quando comparada a participação na tomada de decisão estratégica. 0 relacionamento interpessoal, como variável explicativa de tal variação é apresentada na sequência.

\subsection{Modelo estrutura e hipóteses da pesquisa}

Para análise dos dados pela técnica de Equações Estruturais foram adotados os mesmos procedimentos do estudo de Hall (2008). Para a avaliação da validade de adequação dos construtos, observouse a validade convergente e a validade discriminante. Por meio da validade discriminante verificou-se que todos os indicadores do instrumento de pesquisa apresentaram bons índices de ajuste, com cargas fatoriais acima de 0,5, consideradas por Hair Jr. et al. (2016) como ideais. A matriz Cross Loadings, contendo as cargas fatoriais estão destacadas na Tabela 3.

Tabela 3: Validade Discriminante - Matriz Cross Loadings

\begin{tabular}{lccr}
\hline \multicolumn{1}{c}{ Indicator } & RI & DOP & DES \\
\hline \hline RI1 & 0,8609 & 0,2685 & 0,1800 \\
RI2 & 0,7957 & 0,3605 & $-0,0021$ \\
RI3 & 0,8355 & 0,2212 & 0,1563 \\
RI4 & 0,8496 & 0,3426 & 0,1472
\end{tabular}


Heitor Gerson dos Santos, Joice Denise Schafer \& Rogério João Lunkes.

$\begin{array}{llll}\text { DOP1 } & 0,2550 & 0,8144 & 0,5569 \\ \text { DOP2 } & 0,3398 & 0,8013 & 0,2128 \\ \text { DOP3 } & 0,3269 & 0,8993 & 0,2313 \\ \text { DES1 } & 0,2011 & 0,5962 & 0,5132 \\ \text { DES2 } & 0,1376 & 0,3597 & 0,9683 \\ \text { DES3 } & 0,1041 & 0,2819 & 0,9518\end{array}$

Nota: Rl: relações interpessoais; DOP: Envolvimento na tomada de decisão operacional; DES: envolvimento na tomada de decisão estratégica.

Fonte: Dados da pesquisa

Para confirmar a validade discriminante das variáveis latentes do modelo determinou-se o valor da AVE (Average Variance Extracted). De acordo com Fornell e Larcker (1981), ela é confirmada quando o valor da raiz quadrada da AVE é maior que os valores absolutos das correlações com as demais variáveis latentes. Neste estudo testou-se a validade discriminante pelo critério de Fornell e Larcker (1981). Confirma-se por meio da Tabela 4 que as variáveis satisfazem essa respectiva condição.

Tabela 4: Resultados do modelo de mensuração modelo 1

\begin{tabular}{cccc}
\hline \hline & \multicolumn{2}{l}{ Painel A - Discriminant Validity - Fornell-Larcker Criterion } & \\
\hline \hline Construct & $\mathrm{Rl}$ & $\mathrm{DOP}$ & $\mathrm{DES}$ \\
\hline $\mathrm{RI}$ & 0.6983 & & \\
$\mathrm{DOP}$ & 0.1307 & 0.7022 & 0.6916 \\
$\mathrm{DES}$ & 0.0176 & 0.1321 & \\
\hline \hline
\end{tabular}

Fonte: Dados da pesquisa

Após a análise das cargas fatoriais, realizou-se a apreciação da AVE (Average Variance Extracted) ou variância média extraída (VME) e da Confiabilidade Composta (CC). Na Tabela 5, é destacado ainda 0 Alfa de Cronbach, para enriquecer as análises.

Tabela 5: Resultados do modelo de mensuração modelo 1

Painel B - Construct Reliability/Convergent Validity 
Reflexos das relações interpessoais na participação do controller em decisões organizacionais

\begin{tabular}{cccc}
\hline \hline Construct & Alfa de Cronbach (a) & Confiabilidade Composta & AVE \\
\hline RI & 0.8567 & 0.9025 & 0.6983 \\
DOP & 0.7950 & 0.8757 & 0.7022 \\
DES & 0.7927 & 0.8609 & 0.6916
\end{tabular}

Fonte: Dados da Pesquisa

Observa-se que os três construtos, relação interpessoal, decisão operacional e decisão estratégica apresentaram cargas para a AVE estatisticamente significantes, isto é, com valores iguais ou superiores a 0,50, conforme preconiza Fornell e Larcker (1981). Assim como o alfa de Cronbach excederam o valor de 0,70 e a Confiabilidade Composta (Composite Reliability) apresentou valores maiores que 0,5 . Verifica-se, na validade discriminante, que nenhuma das correlações entre os construtos foi superior a raiz quadrada da AVE, indicando que existe validade discriminante e convergente no modelo proposto.

Para verificar as relações entre os construtos foi utilizada a análise do modelo estrutural. A partir dessa análise confirmou-se a adequação dos modelos de mensuração e atestaram-se a significância das relações entre os construtos do estudo (Hair Jr. et al., 2016). Para avaliar a validade do modelo estrutural foram analisados os seguintes critérios: o tamanho e significância dos coeficientes de caminho e coeficientes de determinação de Pearson $\left(\mathrm{R}^{2}\right)$, obtidos por meio da técnica de Bootstrapping e, ainda, tamanhos do efeito $\left(\mathrm{F}^{2}\right)$ e relevância preditiva obtidos na plataforma blindfolding (Hair Jr. et al., 2016).

Para a execução da técnica de Bootstrapping, para avaliar o nível de significância entre as relações dos construtos, utilizou-se os parâmetros de 5.000 subamostras e 5.000 interações (Hair Jr et al., 2016). Assim, os valores obtidos para a relação entre os construtos são apresentados na Tabela 6. Os efeitos das variáveis de controle são evidenciados na Tabela 7.

Tabela 6: Efeitos entre os construtos do modelo estrutural

\begin{tabular}{rcccc}
\hline \hline Efeitos & Beta & p-value & $F^{2}$ & Hipóteses \\
\hline RI -> DOP & 0,3198 & $0,0291^{* *}$ & 0,1474 & H1 \\
RI -> DES & 0,1384 & 0,2595 & 0,0260 & $H 2$ \\
\hline \hline
\end{tabular}

Nota: ** Significant at $5 \%$.

$R^{2}=$ Decisão operacional $(0,317)$, Decisão estratégica $(0,275)$.

Tabela 7: Efeitos das variáveis de controle

$\begin{array}{llll}\text { Efeitos } & \text { Beta } & \text { p-value } & F^{2}\end{array}$


Heitor Gerson dos Santos, Joice Denise Schafer \& Rogério João Lunkes.

$\begin{array}{llll}\text { Idade -> DOP } & -0,2000 & 0,1680 & 0,0350 \\ \text { Idade -> DES } & -0,1862 & 0,1841 & 0,0286 \\ \text { ExpFunc -> DOP } & 0,1794 & 0,1576 & 0,0309 \\ \text { ExpFunc -> DES } & 0,5443 & 0,0043^{*} & 0,2682 \\ \text { ExpEmp -> DOP } & -0,2437 & 0,0597^{* * *} & 0,0673 \\ \text { ExpEmp -> DES } & -0,0591 & 0,3851 & 0,0037 \\ \text { Genero -> DOP } & 0,3624 & 0,0103^{* *} & 0,1711 \\ \text { Genero -> DES } & 0,2350 & 0,0711^{* * *} & 0,0679\end{array}$

Nota: ${ }^{*}$ Significant at $1 \%,{ }^{* *}$ Significant at $5 \%,{ }^{* *}$ Significant at $10 \%$.

Para as análises dos coeficientes de determinação de Pearson $\left(R^{2}\right)$ o $R^{2}$ entre $2 \%$ e $13 \%$ é entendido como um efeito pequeno, $\mathrm{R}^{2}$ de $13 \%$ a $26 \%$ como efeito médio e $\mathrm{R}^{2}$ acima de $26 \%$ como efeito grande. A partir dos resultados obtidos por meio das Equações Estruturais, verificou-se que o modelo explica $31,7 \%$ do envolvimento do controller na tomada de decisão operacional, quando se trata de decisões estratégicas 0 modelo explica $27,5 \%$. Considera-se, portanto, que há um efeito da variável independente sobre a dependente.

$O \mathrm{~F}^{2}$ é uma medida que avalia se existe um impacto substancial sobre o construto dependente, quando um construto independente é omitido do modelo. Hair Jr. et al. (2016) sugerem a seguinte interpretação para o $F^{2}$ : por efeitos pequenos $(0,02)$, médios $(0,15)$ e grandes $(0,35)$. Desta forma, a omissão das variáveis de controle, experiência do funcionário e gênero, trariam o maior impacto nos construtos de envolvimento do controller na tomada de decisões estratégicas $(0,268)$ e operacionais $(0,171)$, respectivamente.

Com base nos resultados obtidos e apresentados na Tabela 7 é possível inferir estatisticamente que 0 relacionamento interpessoal do controller está positivamente relacionado com a sua participação na tomada de decisão em nível operacional, confirmando, assim, a primeira hipótese da pesquisa. Estes resultados corroboram com Rouwelaar, Bots e Vanamelsfort (2008). De acordo com este estudo, as empresas buscam envolver profissionais com bom relacionamento interpessoal na tomada de decisões operacionais.

O gênero do profissional da controladoria aparece como variável de controle relevante neste contexto. Ao observar as respostas, foi possível observar que existe um maior envolvimento de profissionais do sexo masculino nestas atividades. Chama a atenção, ainda, que a variável de controle "tempo de experiência na empresa" mostrou-se negativamente relacionada ao modelo, o que pode indicar que profissionais com maior tempo nas organizações tendem a desenvolver atividades distintas e serem menos envolvidas na tomada de decisão operacional. Uma hipótese que poderia justificar tais resultados, seria a preferência dos gestores por envolver esse funcionário mais experientes na tomada de decisões estratégicas, aproveitando o conhecimento dele sobre a empresa. Entretanto, essa hipótese não pôde ser confirmada quando analisado o efeito direto do "tempo de experiência na empresa" sobre o envolvimento no controller na tomada de decisão estratégica, como demonstrado na Tabela 7. 
No que se refere ao relacionamento interpessoal e o envolvimento do profissional da controladoria na tomada de decisão estratégica, os resultados não permitiram confirmar a segunda hipótese da pesquisa. No caso da tomada de decisão estratégica, o tempo de experiência do controller na função parece ser a variável mais relevante para definir a sua participação nesse nível de tomada de decisão. Assim como na tomada de decisão operacional, o envolvimento de profissionais do gênero masculino também parece ser maior nas decisões estratégicas.

Cabe destacar que, para confirmar a relevância da variável gênero no envolvimento dos controllers na tomada de decisões organizacionais, aplicou-se o teste não-paramétrico de Mann-Whitney. Com isso, foi possível identificar a inexistência de diferenças significativas entre estes grupos de profissionais no que se refere a idade, experiência na empresa ou na função. Estes resultados indicam que os profissionais, indiferente do gênero, apresentam características similares no que se refere as demais características analisadas. Por fim, o teste confirmou a diferença de médias existentes entre o envolvimento dos controllers do gênero feminino $e$ masculino na tomada de decisão. Enquanto a média do envolvimento de profissionais do gênero masculino na tomada de decisão operacional foi de 4,28, a média da participação de mulheres foi de 3,77 . No que se refere ao envolvimento na tomada de decisão estratégica as médias foram de 3,60 e 3,11, respectivamente.

Os resultados desta pesquisa permitem inferir, portanto, que um bom nível de relacionamento interpessoal entre o controller e sua equipe, bem como com seus pares e superiores, é relevante para que 0 profissional seja envolvido no processo de tomada de decisões operacionais das organizações. Este achado contraria a proposição de Rouwelaar, Bots e Vanamelsfort (2008), de que os profissionais da controladoria participariam da tomada de decisão operacional, indiferentemente de ter ou não um bom relacionamento com os gestores da empresa.

Para que o controller seja envolvido na tomada de decisões de níveis estratégicos, diferente do que ocorre nas decisões operacionais, o relacionamento interpessoal não parece ser decisivo. Embora Weber (2011) e Karlsson (2019) reconheçam a relevância do bom relacionamento interpessoal para a moldagem das tarefas do profissional, aparentemente este fator não é decisivo para o envolvimento do controle na tomada de decisões estratégicas sendo a experiência na função uma variável identificada como maisrelevante no contexto desta pesquisa. Cabe destacar , que conforme Rouwelaar, Bots e De Loo (2018) apenas em algumas empresas o controller é convidado a participar de tomadas de decisões estratégicas, logo outras variáveis relacionadas ao contexto organizacional, ambiental e mesmo a características pessoais da alta administração da empresa também podem justificar o nível de envolvimento do profissional na área de controladoria nas tomadas de decisões de nível estratégico e podem ser investigadas com maior profundidade em agendas futuras.

Dessa forma o estudo contribui com a teoria apontando mais uma variável relevante no contexto da função e envolvimento do controller nas organizações, o relacionamento interpessoal. Na prática, contribui no sentido de demonstrar como o comportamento do controller pode influenciar no seu nível de engajamento na tomada de decisão organizacional, demonstrando que variáveis além de características técnicas são observadas pelos gestores no momento de definir o nível de envolvimento do profissional na tomada de decisão.

\section{Considerações finais}


Diante de um cenário de debate sobre as funções do controller, a presente pesquisa teve como objetivo analisar a influência das relações interpessoais na participação do controller na tomada de decisões organizacionais, em nível operacional e estratégico. Para isso, aplicou-se um questionário junto a profissionais da controladoria de empresas brasileiras. Os 42 respondentes da pesquisa, $90 \%$ possuem mais de 5 anos de experiência na empresa e $70 \%$ mais de 5 anos de experiência como controller. A maior parte destes profissionais são do gênero masculino, formados em Ciências Contábeis e com idade superior a 35 anos.

Os resultados da pesquisa permitem inferir que embora a relação interpessoal esteja positivamente relacionada com a participação do controller na tomada de decisão de questões operacionais, não tem influência sobre a participação do profissional na tomada de decisão estratégica. A experiência do funcionário na empresa, por sua vez, utilizada como variável de controle da pesquisa, mostrou-se altamente relacionada com a participação do controller na tomada de decisões gerenciais, enquanto o gênero aparece como variável de controle relevante para o envolvimento do professional em ambos os níveis de tomada de decisão, demonstrando que controllers do sexo masculino têm maior tendência de serem envolvidos na tomada de decisão organizacional.

Como limitação, destaca-se que neste estudo foram analisados os aspectos relacionais dos controllers para compreender o nível de participação do profissional na tomada de decisão das organizações. Rouwelaar, Bots e De Loo (2018) destacam, no entanto, que além do relacionamento interpessoal do profissional, as características organizacionais e ambientais sãos elementos relevantes para o estudo deste fenômeno. Recomenda-se assim, que estudos futuros busquem identificar estes elementos, bem como evidenciar as contribuições que o profissional da controladoria pode introduzir caso seja envolvido no processo de tomada de decisão das organizações. Estudos futuros podem buscar entender ainda, de que forma a alta gestão das organizações vê a participação deste profissional como membro da equipe responsável pela tomada de decisão, e o perfil que se espera de um controller com tal responsabilidade.

\section{Referências}

Aguiar, E. S., Araújo, L. M. G., Carmo, T. S., \& Soeiro, T. M. (2016). A influência de gênero, idade, formação e experiência nas decisões de investimentos: uma análise do efeito confiança. Revista Evidenciação Contábil \& Finanças, 4(3), 44-55.

Ahmed, A. S., \& Duellman, S. (2013). Managerial overconfidence and accounting conservatism. Journal of Accounting Research, $51(1), 1-30$.

Fernandes, H. N., Thofehrn, M. B., Porto, A. R., Amestoy, S. C., Jacondino, M. B., \& Soares, M. R. (2015). Relacionamento interpessoal no trabalho da equipe multiprofissional de uma unidade de saúde da família. Revista de Pesquisa Cuidado é Fundamental Online, 7(1), 1915-1926.

Ferrari, M. J., Cunha, L. C., Lunkes, R. J., \& Borgert, A. (2013). O perfil do controller sob a ótica do mercado de trabalho brasileiro. Revista de Informação Contábil, 7(3), 25-50.

Fornell, C., \& Larcker, D. F. (1981). Structural equation models with unobservable variables and measurement error: Algebra and statistics.

Gomes, C. V., de Souza, P., \& Lunkes, R. J. (2014). O perfil do profissional da controladoria solicitado por empresas brasileiras. Journal of Globalization, Competitiveness \& Governability/Revista de Globalización, Competitividad y Gobernabilidad/Revista de Globalização, Competitividade e Governabilidade, 8(1), 34-50. 
Goretzki, L., Strauss, E., \& Weber, J. (2013). An institutional perspective on the changes in management accountants' professional role. Management Accounting Research, 24(1), 41-63.

Hair Jr, J. F., Hult, G. T. M., Ringle, C., \& Sarstedt, M. (2016). A primer on partial least squares structural equation modeling (PLSSEM). Sage publications.

Hall, M. (2008). The effect of comprehensive performance measurement systems on role clarity, psychological empowerment and managerial performance. Accounting, Organizations and Society, 33(2-3), 141-163.

Järvenpää, M. (2007). Making business partners: a case study on how management accounting culture was changed. European Accounting Review, 16(1), 99-142.

Karlsson, B., Hersinger, A., \& Kurkkio, M. (2019). Hybrid accountants in the age of the business partner: exploring institutional drivers in a mining company. Journal of Management Control, 30(2), 185-211.

Lavarda, R; Scussel, F; \& Schäfer, J. (2018). The role of the controller in the perspective of strategy as practice: A Theoretical Essay. In: 41st Annual Congress of the European Accounting Association. Milão, 2018.

Lunkes, R. J., \& Schnorrenberger, D. (2015). Estudo das funções do controller solicitadas por empresas na Alemanha. In Anais do Congresso Brasileiro de Custos-ABC.

Lunkes, R. J., Schnorrenberger, D., \& Rosa, F. S. D. (2013). Funções da Controladoria: uma análise no cenário brasileiro. Revista Brasileira de Gestão de Negócios, 15(47), 283-299.

Lunkes, R. J., Schnorrenberger, D., Gasparetto, V., \& Vicente, E. F. R. (2009). Considerações sobre as funções da controladoria nos Estados Unidos, Alemanha e Brasil. Revista Universo Contábil, 5(4), 63-75.

Lunkes, R. J., Schnorrenberger, D., Gasparetto, V., \& Vicente, E. F. R. (2009). Considerações sobre as funções da controladoria nos Estados Unidos, Alemanha e Brasil. Revista Universo Contábil, 5(4), 63-75.

Lunkes, R. J., Schnorrenberger, D., Souza, C. M. D., \& Rosa, F. S. D. (2012). Análise da legitimidade sociopolítica e cognitiva da controladoria no Brasil. Revista Contabilidade \& Finanças, 23(59), 83-101.

Maas, V. S., \& Matejka, M. (2009). Balancing the dual responsibilities of business unit controllers: Field and survey evidence. The Accounting Review, 84(4), 1233-1253.

Marostega, F. L., Lyrio, M. V. L., Lunkes, R. J., \& Schnorrenberger, D. (2014). Análise da produção científica sobre controladoria nas revista brasileiras de contabilidade. Pensar Contábil, 16(59), 10-19.

Massocco, L. N.; Schäfer, J. D.; Lunkes, R. J. Uma Análise do Perfil do Controller Requerido por Empresas em Portugal. Anais do $1^{\circ}$ Congresso de Iniciação Científica de Gestão e Controladoria da Unochapecó, Chapecó, 2016.

Morgeson, F. P., Reider, M. H., \& Campion, M. A. (2005). Selecting individuals in team settings: The importance of social skills, personality characteristics, and teamwork knowledge. Personnel psychology, 58(3), 583-611.

Oro, I. M., Dittadi, J. R., Carpes, A. M. D. S., \& Benoit, A. D. (2009). O perfil do profissional de controladoria sob a óptica do mercado de trabalho brasileiro. Pensar Contábil, 11(44, -15).

Pierce, B., \& O'Dea, T. (2003). Management accounting information and the needs of managers: Perceptions of managers and accountants compared. The British Accounting Review, 35(3), 257-290.

Rouwelaar, H., Bots, J., \& De Loo, I. (2018). The influence of management accountants on managerial decisions. Journal of Applied Accounting Research.

Rouwelaar, J. A. (2007). Theoretical review and framework: The roles of controllers. Available at SSRN 982831.

Rouwelaar, J. A., Bots, J., \& Vanamelsfort, M. (2008). Business unit controller involvement in management: An empirical study in the Netherlands. Available at SSRN 1277863

Schnorrenberger, D., Castro, G. K., \& Lunkes, R. J. (2015). Perspectivas profissionais do controller no Brasil e Espanha. In Anais do Congresso Brasileiro de Custos-ABC.

Siqueira, J. R. M. D., \& Soltelinho, W. (2001). O profissional de controladoria no mercado brasileiro: do surgimento da profissão aos dias atuais. Revista Contabilidade \& Finanças, 12(27), 66-77.

Souza Bido, D., da Silva, D., de Souza, C. A., \& Godoy, A. S. (2010). Mensuração com indicadores formativos nas pesquisas em administração de empresas: Como lidar com a multicolinearidade entre eles?. Administração: Ensino e Pesquisa, 11(2), $245-269$.

Souza, B. C., \& Borinelli, M. L. (2009). As funções de controladoria: um estudo a luz dos anúncios das empresas de recrutamento de profissionais. In Anais do Congresso Brasileiro de Custos-ABC. 
Szukits, Á. (2019). Controllers' profession in contemporary organisations-Evidence from Hungary. JEEMS Journal of East European Management Studies, 24(1), 8-31.

Tzafrir, S. S., \& Eitam-Meilik, M. (2005). The impact of downsizing on trust and employee practices in high tech firms: A longitudinal analysis. The Journal of High Technology Management Research, 16(2), 193-207.

Weber, J. (2011). The development of controller tasks: explaining the nature of controllership and its changes. Journal of Management Control, 22(1), 25-46.

Wolf, S., Weißenberger, B. E., Wehner, M. C., \& Kabst, R. (2015). Controllers as business partners in managerial decision-making. Journal of Accounting \& Organizational Change, 11(1), 24-46.

\section{DADOS DOS AUTORES}

\section{Heitor Gerson dos Santos}

Graduando em ciências contábeis - UFSC

E-mail: heitorg.santos@hotmail.com

\section{Joice Denise Schafer}

Doutoranda em administração - UFSC

Email: schafer.joice@gmail.com

\section{Rogério João Lunkes}

Professor do departamento de ciências contábeis da UFSC

Email: rogeriolunkes@hotmail.com

\section{Contribuição dos Autores:}

\begin{tabular}{|c|c|c|c|}
\hline Contribuição & $\begin{array}{l}\text { Heitor Gerson } \\
\text { dos Santos }\end{array}$ & $\begin{array}{l}\text { Joice D. } \\
\text { Schafer }\end{array}$ & $\begin{array}{l}\text { Rogério J. } \\
\text { Lunkes }\end{array}$ \\
\hline 1. Concepção do assunto e tema da pesquisa & $\mathrm{X}$ & $\mathrm{X}$ & $\mathrm{X}$ \\
\hline 2. Definição do problema de pesquisa & $x$ & $x$ & $x$ \\
\hline \multicolumn{4}{|l|}{$\begin{array}{l}\text { 3. Desenvolvimento das hipóteses e constructos da } \\
\text { pesquisa (trabalhos teórico-empíricos) }\end{array}$} \\
\hline $\begin{array}{l}\text { 4. Desenvolvimento das proposições teóricas } \\
\text { (trabalhos teóricos os ensaios teóricos) }\end{array}$ & & $\mathrm{x}$ & \\
\hline 5. Desenvolvimento da plataforma teórica & $\mathrm{x}$ & & \\
\hline 6. Delineamento dos procedimentos metodológicos & $\mathrm{X}$ & & \\
\hline 7. Processo de coleta de dados & $x$ & & \\
\hline 8. Análises estatísticas & & $\mathrm{X}$ & \\
\hline 9. Análises e interpretações dos dados coletados & $\mathrm{X}$ & & \\
\hline 10. Considerações finais ou conclusões da pesquisa & $\mathrm{x}$ & $\mathrm{x}$ & $x$ \\
\hline 11. Revisão crítica do manuscrito & & & $\mathrm{X}$ \\
\hline 12. Redação do manuscrito & $\mathrm{X}$ & $\mathrm{X}$ & $\mathrm{x}$ \\
\hline
\end{tabular}

